\title{
Effects of Number of Stent Connectors on Mid-Term Vessel Healing and Stent Expansion Assessed by Optical Coherence Tomography
}

\author{
Naotaka Okamoto, MD, ${ }^{1}$ Yutaka Matsuhiro, MD,${ }^{1}$ Akito Kawamura, MD, ${ }^{1}$ \\ Kohei Ukita, MD, ${ }^{1}$ Hitoshi Nakamura, MD,${ }^{1}$ Koji Yasumoto, MD, ${ }^{1}$ Masaki Tsuda, MD, ${ }^{1}$ \\ Akihiro Tanaka, MD, ${ }^{1}$ Yasuharu Matsunaga-Lee, MD,${ }^{1}$ Masamichi Yano, MD, PhD,${ }^{1}$ \\ Yasuyuki Egami, MD, ${ }^{1}$ Ryu Shutta, MD,${ }^{1}$ Yasushi Sakata, MD, PhD, ${ }^{2}$ \\ Jun Tanouchi, MD, $\mathrm{PhD},{ }^{1}$ and Masami Nishino, $\mathrm{MD}, \mathrm{PhD}^{1, *}$ \\ ${ }^{1}$ Division of Cardiology, Osaka Rosai Hospital, Sakai, Osaka, Japan \\ ${ }^{2}$ Department of Cardiovascular Medicine, Osaka University Graduate School of Medicine, Suita, Osaka, Japan
}

\begin{abstract}
Objective: Coronary stents generally consist of hoops and connectors. The number of connectors has been associated with longitudinal strength, flexibility, and deliverability of stents. However, a mid-term impact of number of connectors after stent implantation has been unclear. We sought to examine the effect of number of connectors on vessel healing and stent expansion using optical coherence tomography (OCT).

Materials and Methods: The study included 39 patients with 46 Promus PREMIER stents who underwent OCT examination at 8 months after stent deployment. Promus PREMIER stents have 4 or 5 connectors in a proximal few-millimeter segment of the stents and 2 connectors in the entire stents except the proximal few millimeters. We analyzed OCT cross-sectional images in the proximal 4- or 5-connector segments and adjacent 2-connector segments at $1-\mathrm{mm}$ intervals. OCT parameters including stent apposition, neointimal coverage, the presence of peri-strut low-intensity area (PLIA), stent area, maximal and minimum stent diameters, and stent eccentricity index were compared between the 2 segments.

Results: The 4- or 5-connector segments had a significantly higher proportion of well-apposed struts without neointima (5.8\% [interquartile range (IQR): 0-21.9\%]) compared to the 2-connector segments (0\% [IQR: 0-91.3\%], $\mathrm{p}=0.007$ ). The proportion of struts with PLIA was higher in the 4- or 5-connector segments (7.1\% [IQR: 0-32.0\%]) than in the 2-connector segments ( $0 \%$ [IQR: $0-14.6 \%$ ], $\mathrm{p}=0.026$ ). Stent area, maximal and minimum stent diameters, and stent eccentricity index were comparable between the 2 segments.

Conclusion: The number of connectors were associated with vessel healing processes including neointimal coverage and the presence of PLIA but did not restrict stent expansion.
\end{abstract}

Key words: drug-eluting stents, optical coherence tomography, stent design

\section{Introduction}

Coronary stents generally have a series of hoops and connectors between hoops. Hoops provide radial forces to vessel wall, while connectors link the hoops. Currently, many

\footnotetext{
Division of Cardiology, Osaka Rosai Hospital, 1179-3 Nagasone-Cho, Kita-ku, Sakai, Osaka 591-8025, Japan

E-mail: mnishino@osakah.johas.go.jp

(Received: 2020.December.3; Accepted: 2021. July.27)

doi: 10.15791/angioscopy.oa.20.0037
}

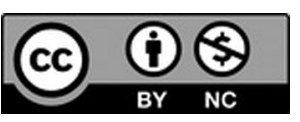

This work is licensed under a Creative Commons [Attribution-NonCommercial 4.0 International] License.

(C) 2021 Japanese Association of Cardioangioscopy kinds of stent platforms are commercially available. Features of the stent platforms are determined based on the number of connectors, strut thickness, stent material, and shape of hoops. For example, stents with a smaller number of connectors have less longitudinal strength, while they have more flexibility, deliverability, conformability to the artery, and easier access to side branches. ${ }^{1,2)}$ Therefore, the number of connectors is important to stent selection during percutaneous coronary intervention. However, it is unclear whether the number of connectors plays a significant role even after stent deployment.

Promus PREMIER (Boston Scientific, Marlborough, MA, USA) stents have a unique design that consists of 4 or 5 connectors in a proximal few-millimeter segment of the stents and 2 connectors in the entire stents except the 


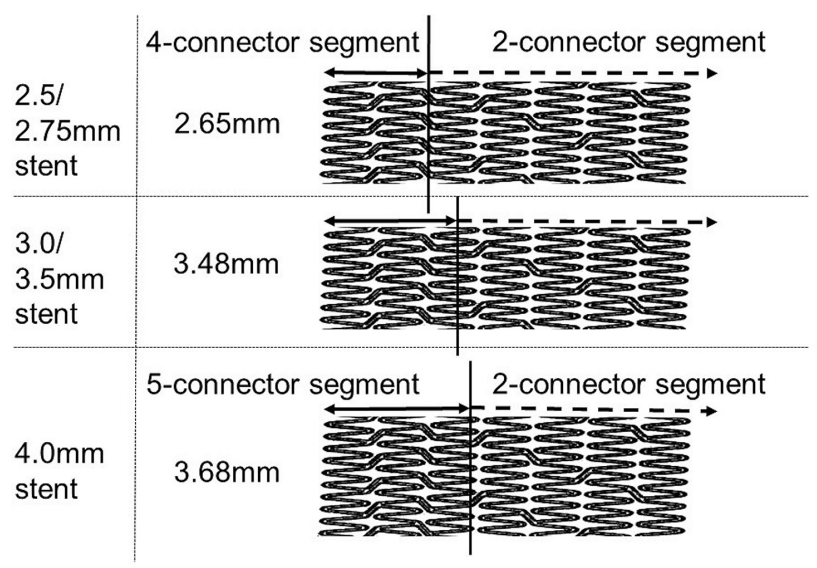

Fig. 1 Stent designs of Promus PREMIER. Promus PREMIER stents have 4 or 5 connectors in a proximal few-millimeter segment of the stents and 2 connectors in the entire stents except the proximal few millimeters. Lengths of the 4- or 5 -connector segments depend on stent diameters.

proximal few millimeters (Fig. 1). The design intends to add longitudinal strength only in the proximal segments where the stent is more likely to be shortened or elongated by device passage and guide catheter compression. ${ }^{3)}$ Optical coherence tomography (OCT) is a coronary imaging device with a high resolution that permits detail vessel and stent analysis. ${ }^{4)}$ The aim of the study was to investigate the impact of the number of connectors on mid-term vessel healing and stent expansion after stent deployment by assessing Promus PREMIER stents using OCT.

\section{Materials and Methods}

\section{Study population}

Routine follow-up coronary angiography and OCT examination at 8 months after stent implantation are performed in our institution, if the patient and stented segments are not unsuitable for OCT examination (e.g. vessel with severe tortuosity, ostium stenosis, patient with chronic kidney disease, and significant restenosis defined as more than $70 \%$ stenosis assessed by angiography). In the study, patients with Promus PREMIER stents were included from our institutional OCT database. This study confirmed to the ethical guidelines outlined in the Declaration of Helsinki.

\section{OCT examination and analysis}

Frequency-domain OCT examinations were performed with a Dragonfly imaging catheter (Abbott Cardiovascular, Plymouth, MN, USA) at 8 months after stent deployment. During acquirement of imaging, nondiluted iodine contrast was injected to achieve blood clearance and auto pull-back system was used. A proximal several-millimeter segment of each stent was analyzed for the study in order to reduce the discrepancy of lesion and procedural characteristics between the 2 segments. Figure 2 shows pictures of Promus PREMIER stents. In the figure, black stars show cross sections analyzed in the 4- or 5-connector segments and white stars present cross sections analyzed in the 2-connector

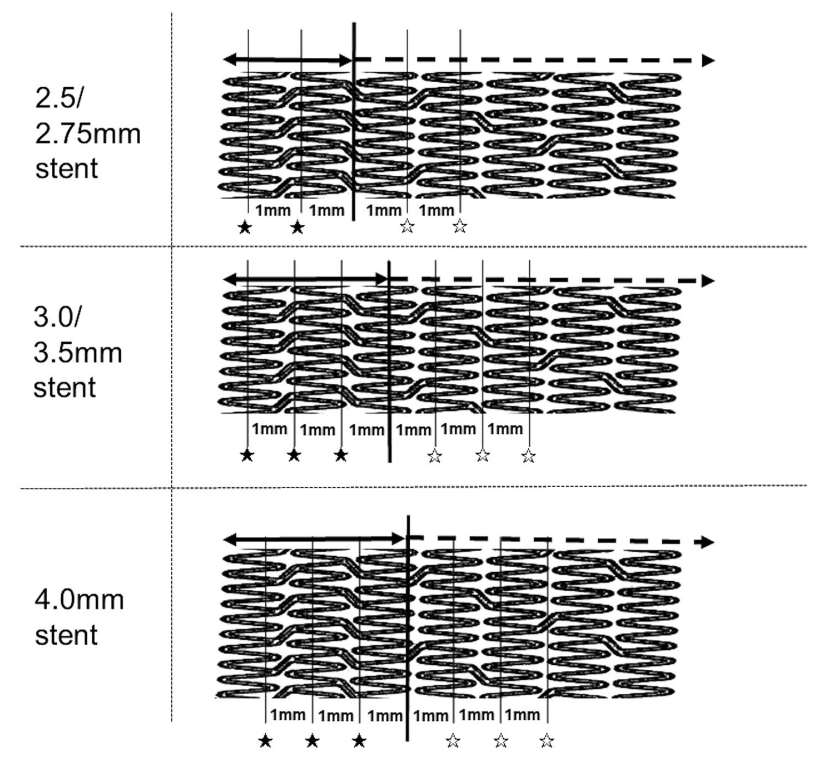

Fig. 2 Cross sections analyzed in each segment. Black stars show cross sections analyzed in the 4- or 5-connector segments and white stars present cross sections analyzed in the 2-connector segments.

segments. In all, 2 cross-sectional OCT images in 4- and 2-connector segments of 2.5- and 2.75-mm stents, and 3 cross-sectional images in 4- or 5- and 2-connector segments of 3.0-, 3.5- and 4.0-mm stents were analyzed at every 1-mm interval, respectively. OCT parameters including average neointimal hyperplasia (NIH) thickness, stent apposition, neointimal coverage, the incidence of peri-strut low-intensity area (PLIA), stent area, lumen area, maximum and minimum stent diameters, and stent eccentricity index (SEI) were compared between the 2 segments. NIH thickness was measured as the distance from the lumen border to the top of strut blooming for each strut. Well-apposed strut was classified as well-apposed strut with neointima if a layer of tissue was visible over all of strut blooming and well-apposed strut without neointima if any part of the strut was visibly exposed to the lumen. Malapposed struts were defined as the struts with $>107 \mu \mathrm{m}$ distance between the top of strut blooming and the lumen border. In all, $107 \mu \mathrm{m}$ was calculated as the sum of strut thickness, polymer thickness, and the OCT resolution limit $(81 \mu \mathrm{m}+8 \mu \mathrm{m}+18 \mu \mathrm{m}=$ $107 \mu \mathrm{m}){ }^{5,6)}$ PLIA was defined as the extent of a region around struts with homogeneous lower intensity appearance than the surrounding tissue without a significant signal attenuation behind the area on OCT images (Fig. 3). ${ }^{7,8)}$ SEI was calculated as the minimum stent diameter divided by the maximum stent diameter.9,10) The values were measured for each cross section and then the average values were calculated for each segment regarding average NIH thickness, stent area, lumen area, maximal and minimum stent diameters, and SEI.

\section{Statistical analysis}

Categorical variables are presented as numbers and percentages. They were compared by either the chi-square test or 


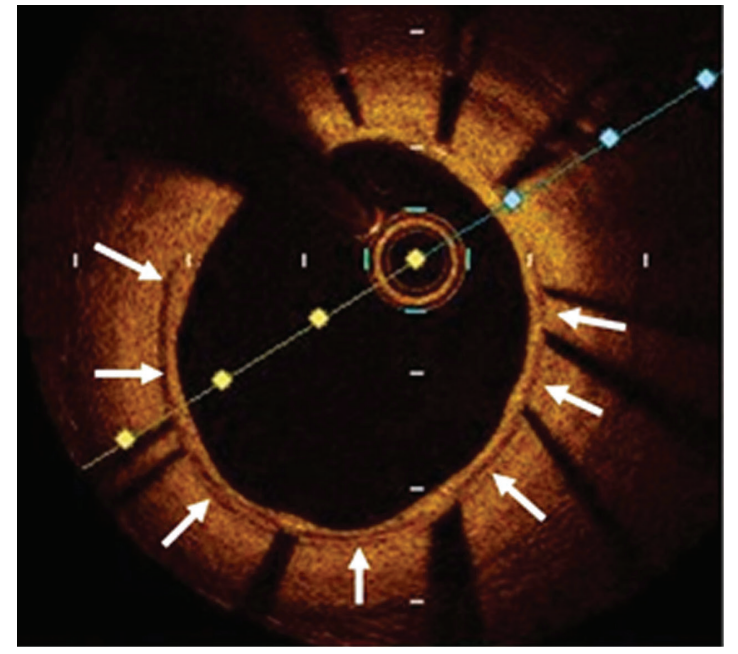

Fig. 3 PLIA. White arrows indicate PLIA. PLIA: peri-strut lowintensity area

the Fisher's exact test as appropriate. Continuous data with normal distribution are presented as means \pm standard deviation and were compared by the t-test. Continuous data without normal distribution are shown as median (25th percentile-75th percentile), and the difference between the 2 segments were calculated by the Kruskal-Wallis test. A value of $\mathrm{p}<0.05$ was considered to be statistically significant. Statistical analyses were performed using JMP version 11.0.0 (SAS Institute Inc., Cary, NC, USA).

\section{Results}

\section{Patient characteristics}

OCT examinations were performed for 39 patients with 46 Promus PREMIER stents and 1135 struts, 116 cross sections in the 4- or 5-connector segments, 1008 struts, and 111 cross sections in the 2-connector segments were analyzed. Patient baseline characteristics are shown in Table 1. The mean age was 66.2 years and male was $74.4 \%$. At follow-up examination, 38 patients $(97.4 \%)$ continued dual antiplatelet therapy and statin was prescribed for 38 patients $(97.4 \%)$. Stent, lesion, and procedural characteristics are presented in Table 2. Mean stent diameter was $3.32 \pm 0.47 \mathrm{~mm}$ and mean stent length was $23.3 \pm 7.8 \mathrm{~mm}$. Half of the lesions was left anterior descending artery. All stents in the study were implanted using intravascular ultrasound (IVUS) guidance. Major stent malapposition those needed additional treatments were not observed by IVUS examination at the end of the procedure.

\section{OCT findings}

The proportion of well-apposed struts with neointima in the 4- or 5-connector segments (91\% [interquartile range (IQR): 75-100\%]) was significantly lower than that in the 2-connecter segments (100\% [IQR: 84-100\%], $\mathrm{p}=0.037)$. On the other hand, the proportion of well-apposed struts without neointima in the 4- or 5-connector segments $(5.8 \%$ [IQR: 0-21.9\%]) was higher compared to that in the
Table 1 Patient characteristics

\begin{tabular}{lc}
\hline Number & 39 \\
Age, years & $66.2 \pm 12.2$ \\
Gender, male & $29(74.4 \%)$ \\
Hypertension & $30(76.9 \%)$ \\
Dyslipidemia & $18(46.2 \%)$ \\
Diabetes mellitus & $18(46.2 \%)$ \\
Smoking & $28(71.8 \%)$ \\
Dialysis & $1(2.6 \%)$ \\
Acute coronary syndrome & $17(44.7 \%)$ \\
Medications & \\
$\quad$ Aspirin & $39(100 \%)$ \\
Clopidogrel & $29(74.4 \%)$ \\
Prasugrel & $9(23.1 \%)$ \\
$\beta$-blocker & $25(64.1 \%)$ \\
ACE inhibitor or ARB & $27(69.2 \%)$ \\
Statin & $38(97.4 \%)$ \\
\hline
\end{tabular}

Values are mean \pm standard deviation or $\mathrm{n}(\%)$.

ACE: angiotensin-converting enzyme; ARB: angiotensin II receptor blocker

Table 2 Stent, lesion, and procedure characteristics

\begin{tabular}{lc}
\hline Number of stents & 46 \\
Number of struts & 2143 \\
Stent characteristics & \\
Stent diameter $(\mathrm{mm})$ & $3.32 \pm 0.47$ \\
Stent length $(\mathrm{mm})$ & $23.3 \pm 7.8$ \\
Lesion characteristics & \\
Lesion vessel & \\
LAD & $23(50.0 \%)$ \\
LCX & $10(21.7 \%)$ \\
RCA & $13(28.3 \%)$ \\
Chronic total occlusion & $2(4.3 \%)$ \\
Intra stent restenosis & $0(0 \%)$ \\
Procedure characteristics & \\
Pre dilation & $16(34.8 \%)$ \\
Post dilation & $22(47.8 \%)$ \\
\hline
\end{tabular}

Values are mean \pm standard deviation or $\mathrm{n}(\%)$.

LAD: left anterior descending artery; LCX: left circumflex; RCA: right coronary artery

2-connector segments (0\% [IQR: 0-91.3\%], $\mathrm{p}=0.007)$. Furthermore, the 4- or 5-connector segments had a higher proportion of struts with PLIA (7.1\% [IQR: 0-32.0\%]) compared to the 2-connector segments (0\% [IQR: 0-14.6\%], $\mathrm{p}=0.026)$. Average NIH thickness was similar in the two segments (46.9 $\mu \mathrm{m}$ [IQR: 27.0-74.9 $\mu \mathrm{m}$ ] in the 4- or 5-connector segments compared to that in the 2-connector segments (55.7 $\mu \mathrm{m}$ [IQR: 29.1-82.2 $\mu \mathrm{m}$ ], $\mathrm{p}=0.410)$. Proportion of malapposed struts was not different between the 2 segments. Stent area was comparable in the 4- or 5-connector segments ( $8.55 \mathrm{~mm}^{2}$ [IQR: 6.42-9.32 $\mathrm{mm}^{2}$ ]) and the 2-connector segments (7.39 $\mathrm{mm}^{2}$ [IQR: 6.40, $\left.\left.9.13 \mathrm{~mm}^{2}\right], \mathrm{p}=0.397\right)$. Maximal and minimum stent 
Table 3 OCT findings

\begin{tabular}{lccc}
\hline & $\begin{array}{c}\text { 4- or 5-connector } \\
\text { segment }\end{array}$ & $\begin{array}{c}\text { 2-connector } \\
\text { segment }\end{array}$ & p value \\
\hline Number of struts & 1135 & 1008 & \\
Average NIH thickness $(\mu \mathrm{m})$ & $46.9(27.0,74.9)$ & $55.7(29.1,82.2)$ & 0.410 \\
$\begin{array}{l}\text { Proportion of well-apposed struts } \\
\text { with neointima (\%) }\end{array}$ & $91(75.0,100)$ & $100(84.0,100)$ & 0.037 \\
Proportion of well-apposed struts & $5.8(0,21.9)$ & $0(0,91.3)$ & 0.007 \\
without neointima $(\%)$ & & & \\
Proportion of malapposed struts $(\%)$ & $0(0,0)$ & $0(0,0)$ & 0.857 \\
Proportion of struts with PLIA $(\%)$ & $7.1(0,32.0)$ & $0(0,14.6)$ & 0.026 \\
Stent area (mm $\left.{ }^{2}\right)$ & $8.55(6.42,9.32)$ & $7.39(6.40,9.13)$ & 0.397 \\
Lumen area $\left(\mathrm{mm}^{2}\right)$ & $7.67(6.18,8.68)$ & $6.81(6.00,8.38)$ & 0.395 \\
Maximal stent diameter $(\mathrm{mm})$ & $3.44(2.98,3.62)$ & $3.31(2.96,3.56)$ & 0.465 \\
Minimum stent diameter $(\mathrm{mm})$ & $3.05(2.76,3.24)$ & $2.92(2.62,3.23)$ & 0.525 \\
SEI & $1.11(1.08,1.15)$ & $1.11(1.08,1.15)$ & 0.634 \\
\hline
\end{tabular}

Values are median (interquartile) or $\mathrm{n}(\%)$.

OCT: optical coherence tomography; NIH: neointimal hyperplasia; PLIA: peri-strut low-intensity area; SEI: stent eccentric index

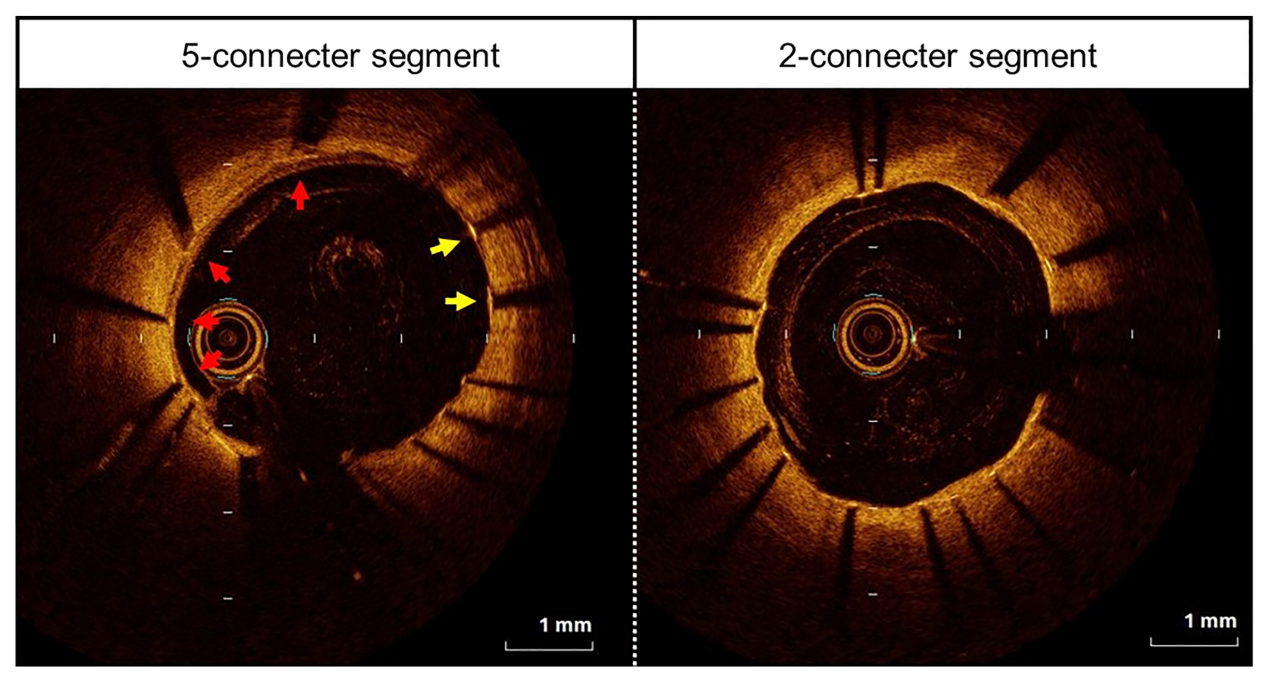

Fig. 4 Representative OCT cross-sectional images of 5- and 2-connecter segments of a 4.0-mm Promus PREMIER stent. Struts with PLIA (red arrows) and well-apposed struts without neointima (yellow arrows) are observed in the 5-connectoer segment (right column). All struts are well apposed with neointima in the 2-connecter segment (left column). OCT: optical coherence tomography; PLIA: peri-strut low-intensity area

diameters and SEI were similar between the 2 segments (Table 3). Representative OCT cross-sectional images of 5and 2-connector segments of a $4.0 \mathrm{~mm}$-Promus PREMIER stent are shown in Fig. 4.

\section{Discussion}

There are several reports comparing vessel healing after stent implantation between different coronary stents, ${ }^{5,11)}$ but the stents have many differences including not only the number of connectors but strut thickness, stent material, shape of hoops, drugs, and polymers. The present study is the first attempt to show the effect of only the number of connectors on mid-term vessel healing and stent expansion using unique design of Promus PREMIER stents. The major findings of the study are as follows. 1) The proportion of well-apposed struts with neointima was significantly lower in the 4- or 5-connector segments than that in the 2-connector segments. On the other hand, the 4- or 5-connector segments had a higher proportion of well-apposed struts without neointima compared to the 2-connector segments. 2) The proportion of struts with PLIA was significantly higher in the 4- or 5-connector segments compared to that in the 2-connector segments. 3) Stent expansion was similar between the 4- or 5-connector and 2-connector segments.

The underlying mechanisms for late stent failure have been investigated using intracoronary imaging modalities 


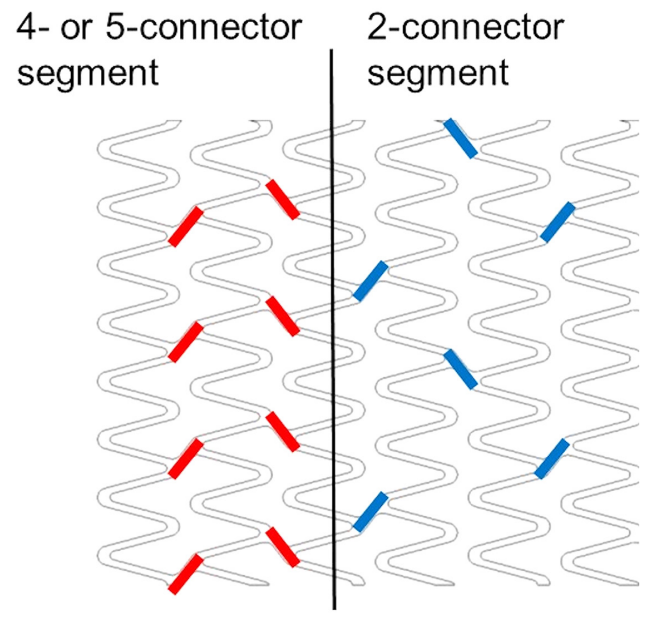

Fig. 5 Number of connectors in each segment. Red lines show the connectors in the 4- or 5-connector segments and blue lines present the connectors in the 2-connector segments. The 4- or 5 -connector segments have a larger amount of metal that is coated with drug than the 2-connector segments.

such as OCT and angioscopy. Previous OCT studies have demonstrated that stent underexpansion, neoatherosclerosis, and stent delayed healing including uncovered and malapposed struts were associated with stent thrombosis. ${ }^{12,13)}$ Ueda et al. reported that in-stent yellow plaque observed by angioscopy at 1 year after stent implantation was an independent risk factor for subsequent stent failure. ${ }^{14)}$ The current study revealed that the number of connectors was related to vessel healing after stent implantation but not associated with stent expansion using OCT.

Drug-eluting stents (DES) release an anti-proliferative drug into the vessel wall to reduce the incidence of occlusive restenosis after stent implantation. The computational study assessing the effect of DES design on drug concentration at the endothelial cell surface has demonstrated that connectors exhibit a relatively high concentration of the drug and stent design with the immediate vicinity of the struts and large fraction of the total surface area occupied by struts presents a high drug concentration. ${ }^{15)}$ The drug concentration should be higher in the 4- or 5-connector segments compared to that in the 2-connector segments, since the 4- or 5-connector segments structurally have more connectors, more immediate proximity of the struts, and larger stent surface area than the 2-connector segments (Fig. 5). The high drug concentration in the 4- or 5-connector segments might lead to the high proportion of well-apposed struts without neointima.

Previous histological studies using a porcine model have demonstrated that PLIA represents the presence of fibrinoid and proteoglycans and is related to inflammation. ${ }^{7,16)}$ In human studies, the presence of PLIA detected by OCT has been considered as a potential marker of abnormal vessel healing, and is associated with neointimal proliferation and an increased incidence of target lesion revascularization. $\left.{ }^{8}, 17\right)$ It has been also reported that minimal connector length in the cross-flow direction and optimal alignment with flow decrease the percentage of in-stent area exposed to low endothelial shear stress (ESS). ${ }^{18)}$ Low ESS promotes inflammation by upregulating various endothelial genes and producing adhesion molecules, chemoattractant chemokines, and pro-inflammatory cytokines. ${ }^{19)}$ In the present study, the 4- or 5-connector segments had a higher proportion of struts with PLIA than the 2-connector segments. The result might be explained by the following reason that the greater number of connectors in the 4- or 5-connector segments led to lager area of low ESS, which induced more inflammation presented as PLIA compared to that in the 2-connector segments. Some struts with PLIA had thick neointima, which is similar to previous studies ${ }^{7,8,16)}$; however, some struts did not have the neointimal coverage probably due to a high drug concentration in the 4- or 5-connector segments. Such heterogeneity of NIH in the 4- or 5-connector segments might result in comparable average NIH thickness compared to that in the 2-connector segments.

Overlapping DES is a widespread practice and is often required for long lesions. The effect of overlapping DES on neointimal coverage assessed by OCT at 9 to 13 months after implantation has been reported to be heterogeneous: on average, DES overlap is associated with more incomplete and thinner neointimal coverage; however in some cases, the overlap elicits an exaggerated neointimal reaction, thicker than in the corresponding nonoverlapping segments. ${ }^{20)}$ The 4- or 5-connector segment has a high metal-to-artery ratio compared to the overlap segment. The process of vessel healing in the 4- or 5-connecter segment might be similar to that in the overlap segment.

Stent area, maximal and minimum stent diameters, and SEI were similar between the 4- or 5-connector and 2 -connector segments. The number of connectors did not restrict stent expansion including area, diameter, and shape.

The new-generation DES have a smaller number of connectors than the first-generation DES. For example, Cypher Select stents (Cordis, Santa Clara, CA, USA) were designed to have 6 connectors for the entire stents. The newgeneration DES have resulted in better clinical outcomes with regard to target lesion failure and stent thrombosis compared to the first-generation DES. ${ }^{21,22)}$ The study showed that stents with a small number of connectors led to good neointimal coverage and decrease in struts with PLIA indicating inflammation. The small number of connectors in the new-generation DES might be one of the underlying mechanisms of the improved clinical outcomes. Furthermore, most of the new-generation DES consist of 2 or 3 connecters and stents with 2 connecters might be better compared to 3-connecter stents from a perspective of the results of this study. However, further exploration is needed to confirm the appropriate number of connecters.

\section{Limitations}

The study was a retrospective single-center study with a small population. Since no baseline OCT examinations were available, the role of number of connectors in immediate results and temporal change of OCT findings were not evaluated. The results need to be interpreted with caution, 
since they were obtained from the analysis of a proximal several-millimeter segment of each stent.

\section{Conclusions}

The large number of connectors was associated with delay in neointimal coverage and increase in struts with PLIA. On the other hand, stent expansion was not related to the number of connectors.

\section{Disclosure Statement}

All authors have no relationships relevant to the contents of this paper to disclose.

\section{References}

1) Ormiston JA, Dixon SR, Webster MW, et al: Stent longitudinal flexibility: a comparison of 13 stent designs before and after balloon expansion. Catheter Cardiovasc Interv 2000; 50: 120-124.

2) Ormiston JA, Webber B, Webster MW: Stent longitudinal integrity bench insights into a clinical problem. JACC Cardiovasc Interv 2011; 4: 1310-1317.

3) Mamas MA, Williams PD: Longitudinal stent deformation: insights on mechanisms, treatments and outcomes from the Food and Drug Administration Manufacturer and User Facility Device Experience database. EuroIntervention 2012; 8: 196-204.

4) Bezerra HG, Costa MA, Guagliumi G, et al: Intracoronary optical coherence tomography: a comprehensive review clinical and research applications. JACC Cardiovasc Interv 2009; 2: 1035-1046.

5) Nakamura D, Lee $Y$, Yoshimura $T$, et al: Different serial changes in the neointimal condition of sirolimus-eluting stents and paclitaxel-eluting stents: an optical coherence tomographic study. EuroIntervention 2014; 10: 924-933.

6) Kubo T, Akasaka T, Kozuma K, et al: Comparison of neointimal coverage between everolimus-eluting stents and sirolimus-eluting stents: an optical coherence tomography substudy of the RESET (Randomized Evaluation of Sirolimus-eluting versus Everolimus-eluting stent Trial). EuroIntervention 2015; 11: 564-571.

7) Teramoto $\mathrm{T}$, Ikeno $\mathrm{F}$, Otake $\mathrm{H}$, et al: Intriguing peri-strut low-intensity area detected by optical coherence tomography after coronary stent deployment. Circ J 2010; 74: 1257-1259.

8) Otake H, Shite J, Ikeno F, et al: Evaluation of the peri-strut low intensity area following sirolimus- and paclitaxel-eluting stents implantation: insights from an optical coherence tomography study in humans. Int J Cardiol 2012; 157: 38-42.

9) Terashita D, Otake H, Shinke T, et al: Differences in Vessel Healing Between Sirolimus- and Everolimus-Eluting Stent Implantation for Bifurcation Lesions: The J-REVERSE Optical Coherence Tomography Substudy. Can J Cardiol 2016; 32: 384-390.

10) Otake H, Shite J, Shinke T, et al: Impact of stent platform of paclitaxel-eluting stents: assessment of neointimal distribution on optical coherence tomography. Circ J 2012; 76: $1880-1888$.

11) Matsuhiro $Y$, Nakamura $D$, Shutta $R$, et al: Difference of vascular healing between bioabsorbable-polymer and durable-polymer new generation drug-eluting stents: an optical coherence tomographic analysis. Int J Cardiovasc Imaging 2021; 37: 1131-1141.

12) Nakamura $D$, Attizzani GF, Toma $C$, et al: Failure Mechanisms and Neoatherosclerosis Patterns in Very Late Drug-Eluting and Bare-Metal Stent Thrombosis. Circ Cardiovasc Interv 2016; 9: e003785.

13) Adriaenssens $T$, Joner M, Godschalk TC, et al: Optical Coherence Tomography Findings in Patients With Coronary Stent Thrombosis: A Report of the PRESTIGE Consortium (Prevention of Late Stent Thrombosis by an Interdisciplinary Global European Effort). Circulation 2017; 136: 1007-1021.

14) Ueda Y, Matsuo K, Nishimoto $Y$, et al: In-Stent Yellow Plaque at 1 Year After Implantation Is Associated With Future Event of Very Late Stent Failure: The DESNOTE Study (Detect the Event of Very late Stent Failure From the Drug-Eluting Stent Not Well Covered by Neointima Determined by Angioscopy). JACC Cardiovasc Interv 2015; 8: 814-821.

15) Seo T, Lafont A, Choi SY, et al: Drug-Eluting Stent Design is a Determinant of Drug Concentration at the Endothelial Cell Surface. Ann Biomed Eng 2016; 44: 302-314.

16) Tellez A, Afari ME, Buszman PP, et al: Peri-strut lowintensity areas in optical coherence tomography correlate with peri-strut inflammation and neointimal proliferation: an in-vivo correlation study in the familial hypercholesterolemic coronary swine model of in-stent restenosis. Coron Artery Dis 2014; 25: 595-601.

17) Kuroda K, Otake H, Shinke T, et al: Peri-strut low-intensity area assessed by midterm follow-up optical coherence tomography may predict target lesion revascularisation after everolimus-eluting stent implantation. EuroIntervention 2019; 14: 1751-1759.

18) Pant S, Bressloff NW, Forrester AI, et al: The influence of strut-connectors in stented vessels: a comparison of pulsatile flow through five coronary stents. Ann Biomed Eng 2010; 38: 1893-1907.

19) Chatzizisis YS, Coskun AU, Jonas M, et al: Role of endothelial shear stress in the natural history of coronary atherosclerosis and vascular remodeling: molecular, cellular, and vascular behavior. J Am Coll Cardiol 2007; 49: 2379-2393.

20) Gutiérrez-Chico JL, Räber L, Regar E, et al: Tissue coverage and neointimal hyperplasia in overlap versus nonoverlap segments of drug-eluting stents 9 to 13 months after implantation: in vivo assessment with optical coherence tomography. Am Heart J 2013; 166: 83-94.

21) Shiomi H, Kozuma K, Morimoto T, et al: Long-term clinical outcomes after everolimus- and sirolimus-eluting coronary stent implantation: final 3-year follow-up of the Randomized Evaluation of Sirolimus-Eluting Versus Everolimus-Eluting Stent Trial. Circ Cardiovasc Interv 2014; 7: 343-354.

22) Toyota T, Shiomi H, Morimoto $T$, et al: Meta-analysis of long-term clinical outcomes of everolimus-eluting stents. Am J Cardiol 2015; 116: 187-194. 